\title{
OPTIMAL INSURANCE AND REINSURANCE POLICIES IN THE RISK PROCESS
}

\author{
BY
}

A.Y. Golubin

\begin{abstract}
The paper examines a classical risk model where both insurance and reinsurance policies are chosen by the insurer in order to minimize the expected maximal loss. We show that the optimal control problem reduces to a static case. We found that the optimal reinsurance is excess of loss reinsurance and describe the set optimal insurance policies. Such a policy providing the minimal variance of the risk left with insured turns out to be a combination of stop loss and deductible policies. The results are illustrated by two numerical examples.
\end{abstract}

\section{KEYWORDS}

Optimal control, risk sharing, insurance, reinsurance.

\section{INTRODUCTION}

The model we start from is a classical risk process

$$
X_{t}=x+c t-\sum_{i=1}^{N_{t}} Y_{i}
$$

where the claim number process $\left\{N_{t}\right\}$ is a Poisson process with a rate $\lambda$ and $\left\{Y_{i}\right\}$ are independent identically distributed claim sizes with probability distribution function $F(x)$ and a finite second moment $E Y^{2}<\infty ;\left\{N_{t}\right\}$ and $\left\{Y_{i}\right\}$ are independent. We denote by $T \leq \infty$ the supremum of the support of $F$, i.e., the supremum of the set of "all possible values" of the claim size $Y$. The premium is calculated by the mean value principle so that the premium rate is $c=(1+\alpha) \lambda E Y$ with a given loading coefficient $\alpha>0$ and, thus, the safety loading condition $c>\lambda E Y$ is ensured.

If the insurer is allowed to reinsure the claims, the problems of optimization over the classes of proportional and excess of loss reinsurance policies were studied, correspondingly, in Schmidli (2001) and in Hipp and Vogt (2003). If the surplus of wealth can be invested into a risky assert, the problem of 
minimizing the ruin probability over the set of investment and proportional reinsurance policies was considered by Schmidli (2002). A model in which investment and insurance policies are chosen by the insured who wishes to maximize the expected utility of consumption was investigated by Gollier (1994).

The principal difference between the presented paper and previous works is that we consider the case where it is possible to choose both insurance and reinsurance policies (investment is not allowed). Criterion to be minimized is the expected maximal losses over the time period $[0, \infty)$. While in the abovementioned problems the tool for deriving an optimal policy is the HamiltonJacobi-Bellman equation that rarely assumes an explicit solution and usually requires a numerical solving, a specific form of the criterion in our setting allows for treatment of the dynamical model without analysis of the HJB equation, by a reduction to a static case. A model close in some sense to ours is that in Waters (1983) (see also Gerber (1980)), where an apriori static problem of maximizing the adjustment coefficient in the Lundberg estimate for the ruin probability was studied. Unlike the presented model, the insurer there chooses only a reinsurance policy, a choice of an insurance policy is not allowed. Note also that we confine ourselves to optimization from the view-point of the insurer, so design of any Pareto-optimal solutions as that in Raviv (1979) or in Golubin (2005) is left beyond the scope.

Now we give a formal description of the model. Suppose the insurer to choose a per-claim insurance of $I_{t}$ and per-claim reinsurance of $A_{t}$. That is, if an insured's claim size at time $t$ is $Y=y$ then he/she is paid $I_{t}(y)$; if the insurer's indemnity payment is $I_{t}(Y)=z$ then the insurer pays $A_{t}(z)$ only, the rest $z-A_{t}(z)$ is covered by the reinsurer. According to the mean value principle, the premium rate left for the insurer is

$$
\begin{aligned}
c_{t}= & \lambda\left\{(1+\alpha) E I_{t}(Y)-\left(1+\alpha_{1}\right) E\left[I_{t}(Y)-A_{t}\left(I_{t}(Y)\right)\right]\right\}= \\
& \lambda\left\{E A_{t}\left(I_{t}(Y)\right)+\alpha_{1}\left[E A_{t}\left(I_{t}(Y)\right)-\delta E I_{t}(Y)\right]\right\},
\end{aligned}
$$

in which $\alpha_{1}>0$ is a reinsurer's loading, $\delta \stackrel{\text { def }}{=}\left(\alpha_{1}-\alpha\right) / \alpha_{1}$ is a relative difference between the reinsurance and insurance loading coefficients. Then the insurer's wealth follows

$$
X_{t}=x+\int_{0}^{t} c_{s} d s-\sum_{i=1}^{N_{t}} A_{t_{i}}\left(I_{t_{i}}\left(Y_{i}\right)\right),
$$

with $\left\{t_{i}\right\}$ denoting the claim times. Allowable insurance and reinsurance policies, $I=\left\{I_{t}\right\}$ and $A=\left\{A_{t}\right\}$, are those measurable and predictable with respect to the information available by $t$, i.e. with respect to the natural filtration $\left\{\mathcal{F}_{t}\right\}$, and such that $0 \leq I_{t}(y) \leq y$ and $0 \leq A_{t}(z) \leq z$. To prevent arbitrage, we assume $\alpha<\alpha_{1}$ since otherwise the insurer can transfer his entire risk to the reinsurer and thus receive a "free lunch" without any uncertainty. 
As a functional to be minimized by the insurer, we consider the expected maximal loss (see for definition, e.g., Bowers et al. (Chap. 12, 1986))

$$
E L=E \sup _{t \geq 0}\left\{\sum_{i=1}^{N_{t}} A_{t_{i}}\left(I_{t_{i}}\left(Y_{i}\right)\right)-x-\int_{0}^{t} c_{s} d s\right\} .
$$

In other words, this is the expectation of the maximum excess of summary loss above summary premium incurred in addition to the initial wealth $x$. From (3), as $\sup _{t \geq 0}\{\cdot\} \geq \sup _{t=0}\{\cdot\}$, we have $L \geq-x$ with probability one, where $x=X_{0}$ is the initial wealth. The least value $E L=-x$ can be achieved simply by putting $I_{t}(y) \equiv 0$, which means $X_{t} \equiv x$ - no insurance contract is made. To avoid this degenerated case, we impose an additional constraint on admissible insurance policies: for each (fixed) claim time $t$,

$$
E I_{t}(Y) \geq M
$$

where $M$ is a given constant such that $0<M<E Y$. In fact, the constraint defines a lower limit $(1+\alpha) \lambda M$ for the premium rate the insurer obtains from insureds.

The optimal control problem we will study is to minimize $E L$ over allowable policies $I$ and $A$ specified above. Since (2) is a controlled Markovian homogeneous process, we can confine consideration to Markov stationary strategies, i.e, at each moment $t=t_{i}$ of making decision the chosen policies $I_{t}$ and $A_{t}$ depend only on the current state $x=X_{t}$, not on the past of the process. Moreover, the value function of this problem is

$$
V(x)=\inf _{I, A} E L
$$

and, in view of (3), $E L=E\left[L \mid X_{0}=0\right]-x$ so that $V(x)=V(0)-x$. Hence, the policies at which the infimum in the right-hand side of (4) is attained do not depend on the current wealth $x$. Therefore, the class of constant policies $I_{t}(y) \equiv I(y)$ and $A_{t}(z) \equiv A(z)$ suffices to solve the problem.

For the case of constant policies, we can use a result in Bowers et al. (1986) derived for the classical process: $E L=E Y^{2} /(2 \alpha E Y)$. In our model this gives the following formula for the expected maximal loss

$$
E L=\frac{E A^{2}(I(Y))}{2 \alpha_{1}[E A(I(Y))-\delta E I(Y)]}-x
$$

where, recall, $\delta=1-\alpha / \alpha_{1} \in(0,1)$. To ensure that $E L$ is well-defined, in addition to the made supposition $E Y^{2}<\infty$ implying $E A^{2}(I(Y))<\infty$, we require $E A(I(Y))>\delta E I(Y)$. As is seen from (1), this inequality is just the safety loading condition for the risk process (2) under the constant policies. Note also that 
the initial wealth $x$ does not have an effect on optimal policies. For convenience, we assume $x=0$ throughout the sequel. Thus, the problem under consideration is the following

$$
\text { minimize } J[I, A]
$$

where an expression for $J[I, A]$ is given by (5) with $x=0$, and minimization is taken over the set of Borel-measurable functions $I(\cdot)$ and $A(\cdot)$ that satisfy: $0 \leq I(y) \leq y, 0 \leq A(z) \leq z, E I(Y) \geq M$, and $E A(I(Y))>\delta E I(Y)$.

Remark in conclusion that throughout the text a coincidence of policies $\left(I^{\prime}, A^{\prime}\right)=\left(I^{\prime \prime}, A^{\prime \prime}\right)$ is understood with probability one, $I^{\prime}(Y)=I^{\prime \prime}(Y)$ and $A^{\prime}\left(I^{\prime}(Y)\right)=A^{\prime \prime}\left(I^{\prime \prime}(Y)\right)$ almost surely (a.s.).

Section 2 deals with two simplified variants of the general problem (6): first, without the possibility of reinsurance and under a constrained premium rate and, second, minimization of $J$ over reinsurance policies only. For the first case we show that the optimal insurance policy is a stop loss policy $I^{*}(y)=$ $y \wedge k^{*}$ having the minimal possible expectation $E I^{*}(Y)=M$ (here $y \wedge x$ denotes $\min \{y, x\})$. In the second problem, the optimal share of risk retained by the insurer, $A^{*}(Y)$, is proved to be a stop loss policy ${ }^{1} A^{*}(Y)=Y \wedge a^{*}$. An optimality condition uniquely determining the retention level $a^{*}$ is derived. In section 3 we study the general case where both insurance and reinsurance are applied. While a unique optimal reinsurance still remains a stop loss policy, the optimal insurance policies constitute a whole class. The structure of this set is described. An interesting point seems that there is a unique optimal insurance policy $I^{*}(y)$ characterized by the least value of the insured's coverage variance. The policy turns out to be a "hybrid" of stop loss and deductible policies and is defined by a pair of parameters. The results of sections 2 and 3 are illustrated by numerical examples involving uniform and exponential distributions of the claim size.

\section{Preliminary Results}

\subsection{Optimization over insurance policies}

Consider a particular case of the above-described model where reinsurance is not allowed or, in our notation, the retention function of the insurer is set to be $A(z) \equiv z$. Then problem (6) takes the form

$$
\text { minimize } J[I] \equiv \frac{E I^{2}(Y)}{2 \alpha E I(Y)} \quad \text { subject to } E I(Y) \geq M \text {. }
$$

\footnotetext{
By the term "stop loss reinsurance policy" we always mean the form of a policy applied to an individual claim, not to aggregate claims. This scheme of reinsurance is usually called excess of loss reinsurance.
} 
Examine first an auxiliary problem with a fixed mean of insurance coverage

$$
\inf _{I: E I(Y)=m} J[I]
$$

where parameter $m \in[M, E Y]$. Since the goal functional is increasing in $E I^{2}(Y)$ under a fixed value of $E I(Y)$, minimization in (8) reduces to minimization of $E I^{2}(Y)$ under the constraint $E I(Y)=m$.

Solution to the latter problem is well known. It is a direct consequence of a result in Ohlin (1969) (see also Kaluszka (2004)) that for a given $M$, the risk sharing $I^{*}(Y)=Y \wedge k$, where $k$ is such that $E I^{*}(Y)=m$, is less risky than any other risk sharing $I(Y)$ with $E I(Y)=m$ in the sense that $E\left[I^{*}(Y)^{2}\right] \leq E\left[I(Y)^{2}\right]$.

We present below another way of proving the optimality of the stop loss policy that employs the Neyman-Pearson lemma. Methodologically, this is justified by that we will further apply the Neyman-Pearson lemma to a more complicated setting of the optimization problem, where both insurance and reinsurance are allowable.

As the functional $E I^{2}(Y)$ is convex in $I$ on the convex set of admissible policies, a policy $I_{m}^{*}$ minimizes $E I^{2}(Y)$ if and only if the derivative

$$
\left.\frac{d}{d \rho} E\left\{\rho I_{m}^{*}(Y)+(1-\rho) I(Y)\right\}^{2}\right|_{\rho=1}=2 \int_{0}^{T} I_{m}^{*}(x)\left[I_{m}^{*}(x)-I(x)\right] d F(x) \leq 0
$$

for any admissible $I$. In other words, $I_{m}^{*}$ is a solution to a problem

$$
\min _{I} \int_{0}^{T} I_{m}^{*}(x) I(x) d F(x) \text { subject to } \int_{0}^{T} I(x) d F(x)=m .
$$

Optimal policies in this kind of optimization problems are characterized by the generalized Neyman-Pearson lemma (see, e.g., Lehmann (1959)). The lemma states that an admissible $I_{m}^{*}$ is optimal if and only if there exists a constant $k$ such that

$$
I_{m}^{*}(y)=\left\{\begin{array}{ll}
0 & \text { if } I_{m}^{*}(y)-k>0 \\
y & \text { if } I_{m}^{*}(y)-k<0
\end{array} \quad \text { up to a set of zero } F\right. \text {-measure. }
$$

As is easily seen, the only function satisfying this condition is $I_{m}^{*}(y)=y \wedge k$ with $k_{d e f}=k_{m}$ uniquely determined by $E[Y \wedge k]=m$, that is, $\int_{0}^{k} \bar{F}(x) d x=m$ where $\bar{F}(x) \stackrel{\text { def }}{=} 1-F(x)$.

Return to problem (7). Apparently,

$$
\inf _{I: E I(Y) \geq M} J[I]=\inf _{m \in[M, E Y]} \inf _{I: E I(Y)=m} J[I] .
$$

Since the inner minimum in the right-hand side is attained at $I_{m}^{*}(y)=y \wedge k_{m}$, we can replace the infimum over $m \in[M, E Y]$ by infimum of $J[I]$ over all 
$I(y)=y \wedge k, k \in\left[k_{M}, T\right]$. Inserting the stop loss policy into expression (7) for $J[I]$, we have $\left.J[I]\right|_{I(y)=y \wedge k}=\int_{0}^{k} x \bar{F}(x) d x /\left\{\alpha \int_{0}^{k} \bar{F}(x) d x\right\}$. Differentiating with respect to $k$ gives that this function is increasing in $k$, therefore the optimal $I^{*}(y)=y \wedge k^{*}$ with $k^{*}=k_{M}$. Thus the following proposition has been proved.

Proposition 1. Problem (7) has a unique solution, a stop loss policy $I^{*}(y)=$ $y \wedge k^{*}$, where the level $k^{*}$ is a unique root on $(0, T)$ of the equation

$$
\int_{0}^{k} \bar{F}(x) d x=M \text {. }
$$

The stop loss form of optimal policy $I^{*}$ is not surprising in view of a result in Bowers et al. (Chap. 13, 1986) where a similar fact was proved for a problem under a constraint $E I(Y)=M$ and with another criterion, the adjustment coefficient of the Lundberg inequality for the ruin probability. A more common insurance arrangement in practice is a deductible policy $I(y)=(y-d)_{+}$. Formally, the deductible policy appears as optimum in a static problem of maximizing the insured's expected utility when the premium is defined by the mean value principle, while the stop loss policy maximizes the expected utility of the insurer (see, e.g., Raviv (1979) or, for a case of a group of insureds, Golubin (2006)). In section 3 we will show that if the insurer has a possibility to use reinsurance also, then there exists an optimal insurance coverage, called an $S D$-policy, of a more "habitual" form that resembles the deductible policy.

Note also that $E I^{*}(Y)$ takes the least possible value equal to $M$. As was noted above, the optimal criterion value $J\left[I^{*}\right]$ increases as $k^{*}$ increases, hence, $J\left[I^{*}\right]$ is increasing with $M$. The same is true for the premium rate $(1+\alpha) \lambda M$. So in a situation when the insurer may choose both $M$ and $I$, the choice can be regarded as a trade-off of the received premium rate against the expected maximum loss under optimal $I^{*}(y)=y \wedge k^{*}$, where $k^{*}=k_{M}$.

\subsection{Optimization by reinsurance}

In this subsection we suppose that risk exchange between the insurer and an insured is not allowed so that the insurer chooses a reinsurance policy only. Then the optimization problem is

$$
\operatorname{minimize} J[A] \equiv \frac{E A^{2}(Y)}{2 \alpha_{1}[E A(Y)-\delta E Y]} \text { subject to } E A(Y)>\delta E Y,
$$

where $\delta=1-\alpha / \alpha_{1}$. Denote by $a_{0} \in(0, T)$ the root of the equation $\int_{0}^{a} \bar{F}(x) d x=$ $\delta E Y$, i.e., the retention level in a stop loss policy under which the denominator in (9) becomes zero. 
Proposition 2. Problem (9) has a unique solution, a stop loss policy

$$
A^{*}(z)= \begin{cases}z \wedge a^{\prime} & \text { if } \psi(T)>0 \\ z & \text { if } \psi(T) \leq 0\end{cases}
$$

where $a^{\prime}$ is a unique root on $\left(a_{0}, T\right)$ of the equation $\psi(a)=0$ with

$$
\psi(a) \stackrel{\operatorname{def}}{=}\left(\int_{0}^{a} \bar{F}(x) d x-\delta E Y\right) a-\int_{0}^{a} x \bar{F}(x) d x .
$$

Proof. Similar to the proof of Proposition 1, first we consider a constrained problem

$$
\text { minimize } J[A] \quad \text { subject to } E A(Y)=m \text {, }
$$

where parameter $m \in(\delta E Y, E Y]$. From (9) it follows that the goal functional is increasing in $E A^{2}(Y)$ under a fixed value of $E A(Y)$. Therefore, by the same reasonings as that in Proposition 1, we show that the only solution to the constrained problem is a stop loss policy. Inserting $A(z)=z \wedge a$ into (9), we obtain the function $\int_{0}^{a} x \bar{F}(x) d x /\left\{\alpha_{1}\left[\int_{0}^{a} \bar{F}(x) d x-\delta E Y\right]\right\}$ on $a \in\left(a_{0}, T\right]$. Differentiating with respect to $a$ gives that the sign of the derivative coincides with the sign of the above-defined $\psi(a)$. Since $\psi\left(a_{0}\right)=-\int_{0}^{a_{0}} x \bar{F}(x) d x<0$ and $\psi^{\prime}(a)=$ $\int_{0}^{a} \bar{F}(x) d x-\delta E Y>0$, condition (10) is necessary and sufficient for optimality.

The optimal reinsurance found in Proposition 2 is an excess of loss reinsurance. This kind of reinsurance coincides with that in the static problem of maximizing the adjustment coefficient (see Waters (1983)) and in the dynamical problem of minimizing the ruin probability in Hipp and Vogt (2003). Of course, due to the differences in the problem settings, the optimality equations determining there a retention level $a^{*}$ differ from (10)-(11).

One can see that as the function in (11) is decreasing in $\delta$, the optimal retention level $a^{\prime}$ increases versus an increase in $\delta=1-\alpha / \alpha_{1}$. When the boundary value $a^{\prime}=T$ is achieved, it means that $A(z) \equiv z$ and the insurer retains the entire risk. Hence, the inequality $\psi(T) \leq 0$ in (10) is equivalent to insurer's refusal to use reinsurance. This inequality can also be rewritten as

$$
T / \alpha_{1} \leq E Y^{2} /(2 \alpha E Y),
$$

where the right-hand side is exactly the expected maximal loss without use of reinsurance. In the case of unbounded support of claim size distribution (e.g., an exponential distribution with $\operatorname{supp} F=[0, \infty)$ so that $T=\infty$ ), we have that the level $a^{\prime}$ in the optimal stop loss policy $A^{*}(z)=z \wedge a^{\prime}$ is always finite, for any shape of the support of $F(x)$. Thus, in this case the insurer always applies for the reinsurance. 
Example 1. Consider the case of exponential distribution $F(x)=1-\exp (-x / \mu)$, $x \geq 0$. Inserting the expression for $F(x)$ into (11), we have $\psi(a)=\mu(a(1-\delta)-$ $\mu(1-\exp [-a / \mu]))$ on $\left(a_{0}, \infty\right)$, where $a_{0}=-\mu \ln (1-\delta)$. Set $\alpha=0.5$, then solving the optimality equation $\psi(a)=0$ yields the results

\begin{tabular}{c|c|c|c|c|c|c}
\hline \hline \multicolumn{7}{c}{$\mu=1$} \\
\hline$\alpha_{1}$ & 0.6 & 0.8 & 1 & 1.2 & 1.4 & 1.6 \\
$a^{*}$ & 0.376 & 1.027 & 1.594 & 2.109 & 2.588 & 3.048 \\
\hline \hline \multicolumn{7}{c}{$\mu=10$} \\
\hline$\alpha_{1}$ & 0.6 & 0.8 & 1 & 1.2 & 1.4 & 1.6 \\
$a^{*}$ & 3.764 & 10.277 & 15.936 & 21.086 & 25.899 & 30.482 \\
\hline \hline
\end{tabular}

The table shows that an increase in the reinsurer's loading $\alpha_{1}$ as well as an increase in $\mu$ leads to a greater value of the (finite) optimal retention level $a^{*}$.

\section{THE GENERAL CASE}

In this section we study problem (6) at full extent, i.e., allowing both insurance and reinsurance policies to be applied (see section 1):

$$
\operatorname{minimize} J[I, A] \equiv \frac{E A^{2}(I(Y))}{2 \alpha_{1}[E A(I(Y))-\delta E I(Y)]}
$$

subject to $0 \leq I(y) \leq y, 0 \leq A(z) \leq z, E I(Y) \geq M$, and $E A(I(Y))>\delta E I(Y)$.

Further, similar to Proposition 2, we will need the notation $a^{0}$ and $a^{1}$ for the roots of the equations $\int_{0}^{a} \bar{F}(x) d x=\delta M$ and $\int_{0}^{a} \bar{F}(x) d x=M$ correspondingly, that can be regarded as retention levels of some stop loss policies of reinsurance.

Theorem 1. A unique optimal reinsurance policy in problem (12) is $A^{*}(z)=z \wedge a^{*}$, where

$$
a^{*}= \begin{cases}a^{1} & \text { if } \varphi\left(a^{1}\right) \leq 0 \\ a^{\prime} & \text { if } \varphi\left(a^{1}\right)>0,\end{cases}
$$

$a^{\prime}$ is the only root on $\left(a^{0}, a^{1}\right)$ of the equation $\varphi(a)=0$ with

$$
\varphi(a)=\left(\int_{0}^{a} \bar{F}(x) d x-\delta M\right) a-\int_{0}^{a} x \bar{F}(x) d x .
$$


The set of optimal insurance policies is constituted by the functions

$$
I^{*}(y)=\left\{\begin{array}{cl}
y & \text { if } y \leq a^{*} \\
a^{*} \leq I^{*}(y) \leq y & \text { if } y>a^{*}
\end{array} \text { satisfying } E I^{*}(Y)=M .\right.
$$

\section{Proof.}

Lemma 1. There exists a solution to problem (12).

The proof is given in Appendix.

Let $\left(I^{*}, A^{*}\right)$ be a solution to (12), that does exist by Lemma 1 . We will show that this implies that the optimal policies take the forms specified in (13)(14). Then, as any such solution gives (see (12)) the same value of the goal functional,

$$
J\left[I^{*}, A^{*}\right]=E\left(Y \wedge a^{*}\right)^{2} /\left\{2 \alpha_{1}\left[E\left(Y \wedge a^{*}\right)-\delta M\right]\right\},
$$

we will obtain the set of all optimal policies.

Policy $A^{*}$ solves the problem $\min J\left[I^{*}, A\right]$, therefore $A^{*}(z)=z \wedge a^{*}$ for an appropriate $a^{*}$ as determined in Proposition 2. Consider now a problem $\min J\left[I, A^{*}\right]$ with an additional constraint $E I(Y)=m^{*}$, where $m^{*}=E I^{*}(Y)\left(\right.$ with $\left.m^{*} \geq M\right)$. Like in the Proposition 1's proof, define $I_{\rho}=\rho I^{*}+(1-\rho) I$ then

$$
\left.\frac{d}{d \rho} J\left[I_{\rho}, A^{*}\right]\right|_{\rho=1} \leq 0
$$

for any admissible $I$ as $I^{*}$ is optimal in the problem above. After differentiating (see (5)), we get the left-hand side of this inequality

$$
\begin{aligned}
& \left.\beta \frac{d}{d \rho} E\left[I_{\rho}(Y) \wedge a^{*}\right]^{2}\right|_{\rho=1}+\left.\gamma \frac{d}{d \rho} E\left[I_{\rho}(Y) \wedge a^{*}\right]\right|_{\rho=1}= \\
& \int_{0}^{T} \mathbf{I}\left\{I^{*}(x)<a^{*}\right\}\left[2 \beta I^{*}(x)+\gamma\right]\left(I^{*}(x)-I(x)\right) d F(x),
\end{aligned}
$$

where $\mathbf{I}\{\cdot\}$ denotes the indicator function, $\beta>0$ and $\gamma<0$ are the partial derivatives of $J$ with respect to $E\left[I_{\rho}(Y) \wedge a^{*}\right]^{2}$ and $E\left[I_{\rho}(Y) \wedge a^{*}\right]$ correspondingly. Thus, $I^{*}$ is a solution to a problem

$$
\begin{gathered}
\operatorname{minimize} \int_{0}^{T} \theta(x) I(x) d F(x) \\
\text { subject to } 0 \leq I(x) \leq x, \int_{0}^{T} I(x) d F(x)=m^{*},
\end{gathered}
$$


where $\theta(x)=\mathbf{I}\left\{I^{*}(x)<a^{*}\right\}\left[2 \beta I^{*}(x)+\gamma\right]$. Applying the generalized NeymanPearson lemma, we have that an admissible $I^{*}$ solves (15)-(16) if and only if there exists a constant $C$ such that

$$
I^{*}(y)= \begin{cases}y & \text { if } \theta(y)<C \\ 0 & \text { if } \theta(y)>C\end{cases}
$$

except, possibly, a set of zero $F$-measure.

In order to determine the value of $C$, suppose at first that $C>0$. Then $\theta(y) \equiv 0$ on $\left[a^{*}, T\right]$ so that $I^{*}(y) \equiv y$ and $m^{*}=E Y$. One can choose another $I$ such that $I(y)=y$ on $\left[0, a^{*}\right], I(y) \geq a^{*}$ on $\left(a^{*}, T\right]$, and $E I(Y)<E Y$. From expression (5) for $J\left[I, A^{*}\right]$, where $A^{*}(I(Y))=Y \wedge a^{*}$, it follows that $J\left[I, A^{*}\right]<J\left[I^{*}, A^{*}\right]$, which gives a contradiction.

Suppose $C \leq \theta(0)(=\gamma<0)$. Then from (17) we have $I^{*}(y) \equiv 0$ (the identity is understood as satisfied for $y \in \operatorname{supp} F$ ) that contradicts the inequality $m^{*}>0$. If $\gamma<C<0$ then, by (17), $I^{*}(y)=y \wedge \tilde{a}$ for some $\tilde{a}$ such that $2 \beta \tilde{a}+\gamma=C$. The function $2 \beta a+\gamma=0.5^{-1} \alpha_{1}\left[E(Y \wedge a)-\delta m^{*}\right]^{-2}\left[2\left(E(Y \wedge a)-\delta m^{*}\right)-E(Y \wedge a)^{2}\right]$ coincides up to a positive multiplier with $\psi(a)$ defined in Proposition 2, where $E Y$ is replaced by $m^{*}$. Therefore $\tilde{a}<a^{*}$. However, $I^{*}(Y) \leq \tilde{a}$ a.s. and, according to Proposition 2, the optimal $a^{*}$ cannot be greater than $\tilde{a}$. The only feasible case here is that $a^{*}$ coincides with the greatest possible value of $I^{*}(Y)$, so that $I^{*}(y)=y \wedge a^{*}$.

If $C=0$ then, by (17) and by that $\theta(y)=C=0$ on $\left[a^{*}, T\right]$, we finally obtain $I^{*}(y)=y$ for $y \leq a^{*}$, and $I^{*}(y) \geq a^{*}$ for $y>a^{*}$.

Show now that the constant $m^{*}\left(=E I^{*}(Y)\right)$ is equal to $M$, the left boundary of admissible values of $E I(Y)$. As was noted above, $A^{*}\left(I^{*}(Y)\right)=Y \wedge a^{*}$ and, hence, $J\left[I^{*}, A^{*}\right]$ is influenced by the form of $I^{*}(y)$ on $\left(a^{*}, T\right]$ through the value $E I^{*}(Y)$ only. Therefore (see (5)), the optimal choice of $I^{*}(y)$ on $\left(a^{*}, T\right]$ must minimize $E I^{*}(Y)$ subject to $I^{*}(y) \geq a^{*}$ and $E I^{*}(Y) \geq M$. To prove that this minimal value is $M$, suppose at first that $a^{*}>a^{1}$. Hence $I^{*}(y) \equiv y \wedge a^{*}$ and $E I^{*}(Y)>M$. On the other hand, since $I^{*}(y) \equiv A^{*}\left(I^{*}(y)\right)$, the insurer can, by Proposition 1, choose a better $a: \int_{0}^{a} \bar{F}(x) d x=M$, therefore $a^{*} \leq a^{1}$. Then the optimal choice of the function $I^{*}(y)$ on $\left(a^{*}, T\right]$ must give $E I^{*}(Y)=M$. Note that the degenerated case $a^{*}=a^{1}$ means $I^{*}(y) \equiv y \wedge a^{1}$.

Applying Proposition 2, we obtain that the optimal level $a^{*} \leq a^{1}$ is uniquely determined by (13). Further, any function $I^{*}(y)$ from the set (14) coupled with $A^{*}(z)=z \wedge a^{*}$ gives the same optimal value $J\left[I^{*}, A^{*}\right]$. Thus (13)-(14) determine the set of all solutions to (12).

Theorem 1 states, in particular, that after determining the retention level $a^{*}$, the insurer has typically an infinite set of optimal insurance policies defined in (14). He is indifferent to a choice among them - each such a policy $I^{*}$ gives the same optimal value $J\left[I^{*}, A^{*}\right]$. To proceed with analysis of problem (12), we 


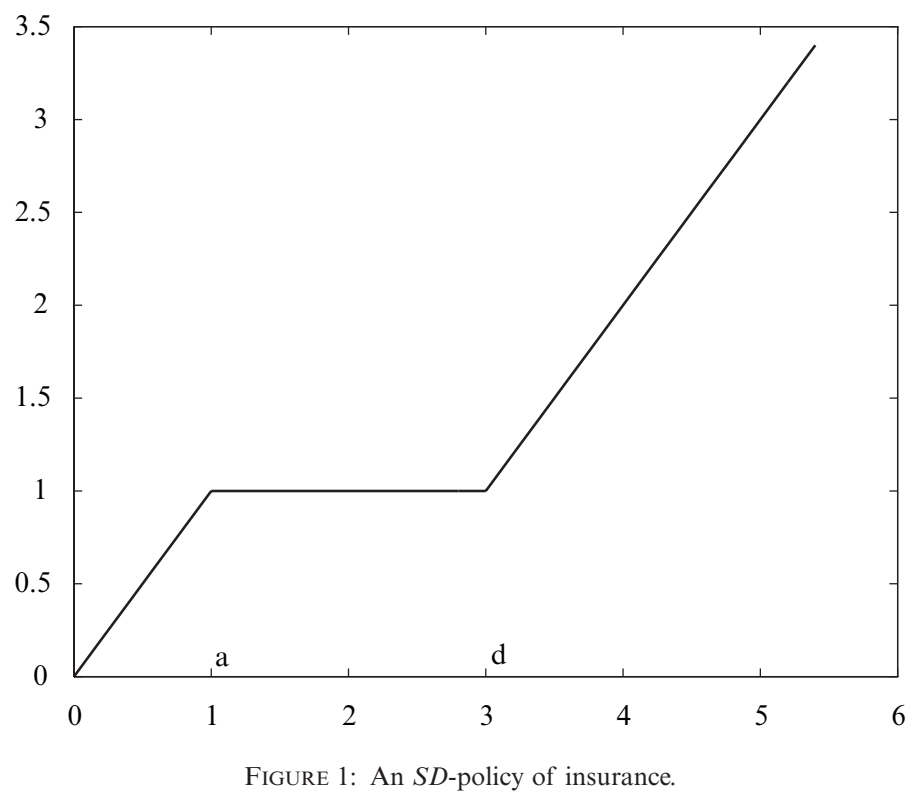

introduce a kind of insurance policies that, as we will see, turns out to be the "best" from a view-point of the insured among all the insurance policies $I^{*}$ optimal in (12). A two-parameter policy

$$
I^{a, d}(y)= \begin{cases}y \wedge a & \text { if } y<d \\ y-(d-a) & \text { if } y \geq d\end{cases}
$$

where $0 \leq a \leq d$, will be called an $S D$-policy. In other words, it is a combination of a stop loss policy $y \wedge a$ and a deductible policy $(y-d)_{+}$. Such a policy with $a=1$ and $d=3$ is presented on Fig. 1. It is seen that an $S D$-policy may be regarded as a generalization, in some sense, of a deductible policy in which, however, insurance payment up to level $d$ is not zero but a stop loss payment. Under the introduced policy, the insurer takes the "tail" of claim size distribution and leaves a "medium" share of insured's risk $Y-I^{a, d}(Y)=(Y-a)_{+} \wedge(d-a)$ with the insured. From the view-point of a potential policyholder, this kind of insurance seems more attractive than the stop loss insurance obtained in Proposition 1 , where the insurer pays all small claims and pays only a threshold $k$ for large claims.

Corollary 1. A unique insurance policy $I^{*}$, which is optimal in (12) and provides a minimal variance of the risk $W^{\text {def }}=Y-I^{*}(Y)$ covered by the insured himself, is 
an SD-policy $I^{a^{*}, d^{*}}(y)$, where $a^{*}$ is given by (13) and $d^{*}$ is the only root of the equation

$$
\int_{0}^{a^{*}} \bar{F}(x) d x+\int_{d}^{T} \bar{F}(x) d x=M .
$$

Proof. Fix any optimal policy $I^{*}$, then the corresponding insured's coverage is $W(Y)=Y-I^{*}(Y)=\left\{\begin{array}{cl}0 & \text { if } Y \leq a^{*} \\ Y-I^{*}(Y) & \text { if } Y>a^{*}\end{array}\right.$ as follows from (14). Minimization of the variance $\operatorname{Var} W(Y)=E W^{2}(Y)-[E W(Y)]^{2}$ reduces to minimization of the second moment $E W^{2}(Y)$ as the mean value is given, $E W(Y)=E Y-M$. This leads to a problem

$$
\min _{I} \int_{a^{*}}^{T} W^{2}(x) d F(x) \text { subject to } \int_{a^{*}}^{T} W(x) d F(x)=E Y-M .
$$

Here, by the definition of $W(y)$ and definition of the set of $I^{*}(y)$ in (14), minimization is taken over the functions $W(\cdot)$ that satisfy $0 \leq W(y) \leq y-a^{*}$ on $y \in\left[a^{*}, T\right]$. As before, applying the generalized Neyman-Pearson lemma, we have that the only solution to the problem is $\hat{W}(y)=\left(y-a^{*}\right) \wedge\left(d^{*}-a^{*}\right)$, where the level $d^{*}\left(\geq a^{*}\right)$ is determined by $E \hat{W}(Y)=E Y-M$. Recall that $W(y)=y-$ $I^{*}(y)$, then the solution found can be rewritten in terms of insurance policy as $\hat{I}(y)=y-\hat{W}(y)=\left(y-\left(d^{*}-a^{*}\right)\right)_{+}$on $y \in\left[a^{*}, T\right]$. Since any optimal insurance policy equals $y$ on $\left[0, a^{*}\right]$, we can extend $\hat{I}$ on the whole interval $[0, T]$ and thus obtain the $S D$-policy $I^{a^{*}}, d^{*}(y)$ indicated in the statement of Corollary 1. The equation $E \hat{W}(Y)=E Y-M$ uniquely determining $d^{*}$ can clearly be put as $E I^{a^{*}, d}(Y)=M$ which coincides with equation (18).

When the pair of optimal policies $\left(I^{a^{*}, d^{*}}, A^{*}\right)$ are applied, the reinsurer's indemnity $I^{a^{*}, d^{*}}(Y)-I^{a^{*}, d^{*}}(Y) \wedge a^{*}=\left(Y-d^{*}\right)_{+}$is of the same form as his indemnity $\left(Y-a^{\prime}\right)_{+}$in the problem without insurance optimization in subsection 2.2. As stated in Corollary 1, the particular choice by the insurer of $I^{a^{*}}, d^{*}$ as an optimal insurance policy makes the related insured's risk $\hat{W}=Y-I^{a^{*}, d^{*}}(Y)$ more attractive for insured, since the variance $\operatorname{Var} \hat{W}$ is the least among values $\operatorname{Var} W$ when $I^{*}$ runs over the set of all optimal insurance policies. The inequality $\varphi\left(a^{1}\right) \leq 0$ in (13) plays the same role as the inequality $\psi(T) \leq 0$ in Proposition 2: it means that the reinsurer's loading $\alpha_{1}$ is large enough for the insurer to refuse reinsurance and retain his entire risk, which is in this case $I^{*}(Y)=Y \wedge a_{1}$ because $d^{*}=T$ by (18).

Remark also that, like in the choice of insurance policy only (Proposition 1), the expected insurer's indemnity (without regard to reinsurance) takes the minimum of possible values, $E I^{*}(Y)=M$.

Example 2. Here $F(x)$ is the uniform distribution on $[0,1]$, so that $T=1$ and $E Y=0.5(>M)$. To determine a solution to problem (12), first we find the 
retention level $a^{*}$. The function $\varphi(a)$ takes the form $a\left(a / 2-a^{2} / 6-\delta M\right)$ on $a \in$ $\left(a^{0}, a^{1}\right)$, where the boundary points $a^{0}=1-\sqrt{1-2 \delta M}$ and $a^{1}=1-\sqrt{1-2 M}$ (recall that $\delta=1-\alpha / \alpha_{1}$ ). Therefore from (13) we have

$$
a^{*}= \begin{cases}1-\sqrt{1-2 M} & \text { if } \delta \geq(1+2 M-\sqrt{1-2 M}) /(6 M) \\ 1.5(1-\sqrt{1-8 \delta M / 3}) & \text { otherwise }\end{cases}
$$

Solving equation (18) with respect to $d$ gives $d^{*}=1-\sqrt{\left(1-\alpha^{*}\right)^{2}+2 M-1}$. Thereby, the optimal stop loss policy $A^{*}(z)=z \wedge a^{*}$ and $S D$-policy $I^{*}(y)=$ $I^{a^{*}, d^{*}}(y)$ are completely defined. The table below presents numerical results for a case of a fixed insurer's loading $\alpha=0.5$.

\begin{tabular}{l|c|c|c|c|c|c|c|c}
\hline \hline & \multicolumn{4}{|c|}{$M=0.25$} & \multicolumn{4}{c}{$M=0.4$} \\
\hline$\alpha_{1}$ & 0.6 & 0.8 & 1 & 1.2 & 0.6 & 0.8 & 1 & 1.2 \\
$a^{*}$ & .086 & .201 & .275 & .293 & .139 & .338 & .475 & .553 \\
$d^{*}$ & .421 & .628 & .839 & 1 & .264 & .512 & .725 & 1 \\
$E L$ & .143 & .251 & .275 & .276 & .233 & .423 & .475 & .482 \\
\hline \hline
\end{tabular}

The critical value $\delta=(1+2 M-\sqrt{1-2 M}) /(6 M)$ in the case $M=0.25$ corresponds to reinsurer's loading $\alpha_{1}^{\prime}=1.06$ under which the function $\varphi(a)$ becomes positive when $a$ passes through $a^{1}=0.293$ (see (13)). For any $\alpha_{1} \geq 1.06$ (see the fifth column), $a^{*}$ and $d^{*}$ remains equal to $a^{1}=0.293$ and 1 . This situation means rejection of reinsurance, the optimal insurance is then a stop loss policy $\left.I^{a^{*}, d^{*}}(y)\right|_{d^{*}=1}=y \wedge 0.293$. In the case $M=0.4$, the related figures are $\alpha_{1}^{\prime}=$ 1.145 and $a^{1}=0.553$ so that $a^{*}=0.553$ and $d^{*}=1$ for any $\alpha_{1} \geq 1.145$. One can also see that a growth of $M$, the low limit of values of $E I(Y)$, increases the first retention level $a^{*}$ and has an opposite effect on $d^{*}$. The reinsurer's coverage of the claim $\left.I^{a^{*}, d^{*}}(Y)-I^{a^{*}, d^{*}}(Y) \wedge a^{*}\right)=\left(Y-d^{*}\right)_{+}$increases since the level $d^{*}$ lessens.

\section{Remark 1.}

Concerning a direction for future research, it seems interesting to study similar insurance and reinsurance optimization problems with other goal functionals. Such a criterion may be the ruin probability as in Schmidli (2002), or the expected discounted utility of consumption as in Gollier (1994), or the expected utility of risk exchange in a static setting similar to Golubin (2002). It is believed (but has not been proved yet) that optimal reinsurance and insurance policies will be still of the forms $A^{*}(z)$ and $I^{*}(y)$ indicated above (i.e., a stop loss policy and an $S D$-policy), provided that:

(i) the decision maker choosing insurance and reinsurance policies is the insurer, a choice among the set of his optimal policies is made by the insured; 
(ii) insurance premium (possibly, under a constraint as in our setting) and reinsurance premium are defined by the mean value principle.

Of course, the parameters $a^{*}$ and $d^{*}$ in the policies $A^{*}$ and $I^{*}$ may now depend on the current wealth $X_{t}$ and, in a non-stationary model, on the time $t$. Optimality equations for the parameters may be quite different from those in Theorem 1 and Corollary 1, but the very forms of optimal policies will perhaps remain the same.

\section{APPENDIX}

\section{Proof of Lemma 1.}

Denote by $\Pi$ the set of admissible policies $(I, A)$ in (12). Let $\left\{I_{n}, A_{n}\right\} \subset \Pi$ be a sequence minimizing (12), that is, $\lim _{n \rightarrow \infty} J\left[I_{n}, A_{n}\right]=J^{*} \stackrel{\text { def }}{=} \inf _{(I, A) \in \Pi} J[I, A]$. For example, the sequence $\left\{I_{n}, A_{n}\right\}$ can be chosen such that $J\left[I_{n}, A_{n}\right] \leq J^{*}+1 / n$. Show first that the denominator in (12) is bounded away from zero: there exists $\varepsilon>0$ such that $E A_{n}\left(I_{n}(Y)\right)-\delta E I_{n}(Y) \geq \varepsilon$ for all $n$. Suppose the contrary then, by the same reasonings as in the Proposition 1's proof, we have $E A_{n}^{2}\left(I_{n}(Y)\right) \geq$ $E\left[I_{n}(Y) \wedge a^{\prime}\right]$ for some $a^{\prime}>0$ and all $n$. The numerator in (12) is thus bounded away from zero uniformly in $n$. Hence, the supposed convergence $E A_{n}\left(I_{n}(Y)\right)-$ $\delta E I_{n}(Y) \downarrow 0$ contradicts the minimizing property of $\left\{I_{n}, A_{n}\right\}$.

By Helly's theorem, there exists a subsequence $\left\{I_{k}(Y), A_{k}\left(I_{k}(Y)\right)\right\}$ weakly converging to a pair $(\xi, \eta)$. To prove that $\xi$ and $\eta$ are proper stochastic values, note that $I_{k}(Y) \leq Y$ and $A_{k}\left(I_{k}(Y)\right) \leq Y$ a.s. Hence, the corresponding distribution functions $F_{k}^{I}(x) \geq F(x)$ and $F_{k}^{A}(x) \geq F(x)$, therefore the limits $\xi$ and $\eta$ are such that $P\{\xi<\infty, \eta<\infty\}=1$. Since $\xi$ is measurable with respect to a sigmaalgebra $\sigma(Y)$ generated by $Y$ and $0 \leq \xi \leq Y$ a.s., the limit $\xi$ can be represented as $\xi=I^{*}(Y)$ for some admissible indemnity function $I^{*}(y)$. Analogously, the other limit $\eta$ can be represented as $\eta=A^{*}\left(I^{*}(Y)\right)$ for some admissible reinsurance policy $A^{*}(z)$. Finally, the fact that $J^{*}=J\left[I^{*}, A^{*}\right]$ follows from the proved weak convergence and finiteness of the expectations $E I^{*}(Y), E A^{*}\left(I^{*}(Y)\right)$, and $E\left[A^{*}\left(I^{*}(Y)\right)^{2}\right]$.

\section{REFERENCES}

Bowers, N.L., Gerber, H.U., Hickman, J.C., Jones, D.A. and Nesbitt, C.J. (1986) Actuarial Mathematics. The Society of Actuaries, Itaca, Illinois.

Gerber, H.U. (1980) Principles of Premium Calculation and Reinsurance. In: Transactions of 21st Int. Congr. of Actuaries, 1, 137-142.

Gollier, C. (1994) Insurance and Precautionary Capital Accumulation in a Continuous-Time Model. Journal of Risk and Insurance, 61, 78-95. 
Golubin, A.Y. (2002) Franchise Optimization in the Static Insurance Model. Journal of Mathematical Sciences, 112, 4126-4140.

Golubin, A.Y. (2005) Pareto-optimal Contracts in an Insurance Market. ASTIN Bulletin, 35(2), 363-378.

Golubin, A.Y. (2006) An Optimal Insurance Policy in the Individual Risk Model Seen as a Bargaining Game. Game Theory and Applications, 11, 60-71.

HiPP, C. and Vogt, M. (2003) Optimal Dynamical XL Reinsurance. ASTIN Bulletin, 33, 193-207.

Kaluszka, M. (2004) Mean-Variance Optimal Reinsurance Arrangements Scand. Actuarial J., 1, 28-41.

Lehmann, E.L. (1959) Testing Statistical Hypotheses. Wiley and Sons, New York.

OHLIN, J. (1969) On a class of measures of dispersion with application to optimal insurance. ASTIN Bulletin, 5, 22-26.

Raviv, A. (1979) The Design of an Optimal Insurance Policy. The American Economic Review, 69, 84-96.

Schmidli, H. (2001) Optimal Proportional Reinsurance Policies in a Dynamic Setting. Scand. Actuarial J., 55-68.

Schmidl, H. (2002) On Minimizing the Ruin Probability by Investment and Reinsurance. Annals of Applied Probability, 12, 890-907.

WAters, H.R. (1983) Some Mathematical Aspects of Reinsurance. Insurance: Mathematics and Economics, 2, 17-26.

Golubin A.Y.

Russian Academy of Sciences

Design Information Technologies Center,

Marshala Zhukova Street, 30-A

Odintsovo

Moscow region, 143000

Russia

E-Mail: io@miem.edu.ru info@ditc.ras.ru 